\title{
Quality Assurance (QA) Assessment for Malaria Rapid Diagnostic Test in Ngoma District, Eastern Province of Rwanda: A cross-sectional prospective study.
}

Mr. Metuschelah HABIMANA, MSc Field Epi ( $\sim$ hmetuschelah@yahoo.com )

Rwinkwavu hospital https://orcid.org/0000-0001-8137-1708

Dr. Vedaste NDAHINDWA, MD, MSc

University of Rwanda School of public health

Mrs. UMUHOZA Stella Matutina, MSc

University of Rwanda School of public health

Dr. Jared OMOLO, MD, MSc

CDC Rwanda\& University of Rwanda, School of Public health, Field Epidemiology and Laboratory Training Program

Mrs. Schifra UWAMUNGU, MSc

University of Rwanda School of public health

Dr. Lucchi Naomi W., Ph.D

CDC/Rwanda

Dr. James HUMUZA, (MD, MSc, PhD)

University of Rwanda School of public health

Research Article

Keywords: Quality assurance, Malaria rapid diagnostic test, Microscopy, Sensitivity, Specificity, Accuracy

Posted Date: November 30th, 2021

DOI: https://doi.org/10.21203/rs.3.rs-1125921/v1

License: (9) (7) This work is licensed under a Creative Commons Attribution 4.0 International License. Read Full License 


\section{Abstract \\ Background}

Currently, malaria rapid diagnostic tests (mRDTs) are increasingly used for diagnosis of malaria, particularly in community where microscopy-based diagnosis is not practical. However, the diagnostic accuracy of mRDTs performed by the community health workers (CHWs) remains unknown. This study was conducted to determine the accuracy of mRDT results performed by CHWs in Ngoma district, eastern province of Rwanda.

\section{Method}

This was a cross sectional prospective study. 420 blood samples of patients self-reported to CHWs for malaria diagnosis were collected and analyzed by CHWs using mRDT and quality control tests were performed by using microscopy as a reference test. The study was conducted from 22nd April to 08th July, 2021.

\section{Results}

Among the 420 patients, 234 (55.71\%) were females and 186 (44.29\%) were males. Malaria test positivity was $2.62 \%$ by using mRDT and $1.67 \%$ by using microscopic test. The sensitivity and specificity of mRDT were $85.71 \%$ and $98.78 \%$ respectively. Negative predictive value, positive predictive value and accuracy of mRDTs were $99.75 \%, 54.54 \%$ and $98.57 \%$ respectively. Sensitivity of mRDT was below the WHO recommended sensitivity (>95\%) although the specificity (98.78\%) was within the WHO recommended specificity ( $>=90)$. There was a substantial agreement between mRDT and malaria microscopic test results, $\mathrm{k}=0.642$.

\section{Conclusion}

mRDTs continue to be an appropriate choice for malaria diagnosis in the absence of microscopy.

\section{Introduction}

Internationally, malaria is a major public health problem. In 2014, the World Health Organization (WHO) reported that on average 3.3 billion people were at risk of malaria (1). In 2020, the WHO indicated that 229 million cases of malaria occurred worldwide, and 215 million cases of malaria (94\%) occurred in Africa, and globally 409000 deaths occurred due to malaria in 2019(2). Eastern and southern provinces are the most vulnerable regions to malaria in Rwanda. The number of cases tripled in the eastern province (from 460,460 in 2013-2014 to 1.4 million in 2015- 2016), and doubled in the Southern province (from 554,035 in 2013-2014 to over 1.1 million in 2015- 2016 (3). The highest prevalence of malaria in Rwanda was observed in eastern part and estimated to be $11.11 \%(4)$. WHO recommend parasitological confirmation through microscopy or malaria rapid diagnostic test (mRDT) for each suspected patient (5), and WHO recommended that all mRDTs sensitivity (compared to microscopy as the "gold standard") should be above $95 \%$ and a minimum specificity of at least $90 \%$ for all malaria species(6)(7). mRDTs accounted for 71\% of the diagnostic tests performed in sub-Saharan Africa in 2014(8). A study conducted between 2009 and 2015 in 19 sub-Saharan countries revealed that the prevalence of malaria using microscopy test was $24.4 \%$ while the prevalence of malaria using mRDT was found to be $30.3 \%(9)$. During a study conducted in Nigeria for evaluating and comparing the performance of the microscopy as gold standard test with other four malaria rapid diagnostic test (mRDT) kits (carestart, SB Bioline, LabAcon and Global kits), their respective sensitivity of mRDTs were $83.7 \%, 86.5 \%, 84.9 \%$ and $86.5 \%$, while their respective specificities were $96.00 \%, 95.80 \%, 95.30 \%$ and $95.40 \%$. it was concluded that those mRDT kits could not replace microscopy(10). Another study carried out in 2020 in Nigeria aimed at comparing the sensitivity and specificity of mRDTs (carestart TM) with the microscopy as gold standard, it was found that mRDTs had a sensitivity and specificity of $29 \%$ and $89 \%$ respectively. These low values of sensitivity were significantly associated with parasite density $(\mathrm{pv}<0.001)$ and other mRDTs kit that could detect malaria parasites at low density were recommended (11). Another similar study conducted in Nigeria for assessing the performance of mRDTs in febrile under-five children at a tertiary health facility level, demonstrated that mRDTs' sensitivity, specificity, positive and negative predictive values were respectively $40.3 \%, 89.6 \%, 81.8 \%$, and $56.5 \%$, respectively and it was recommended that febrile children with positive mRDT results would be confirmed as having malaria while those with negative mRDT results would be retested again using microscopy (12). Another study conducted in sierra Leone in 2020 revealed different prevalence of malaria in under-five children by using mRDTs and microscopy ( $52.67 \%$ and $40.05 \%$ respectively) and the overall sensitivity and specificity of mRDTs were $85.52 \%$ and $69.23 \%$ respectively and these values were lower than the values recommended by WHO (95\% and 90\% respectively)(13)(6). Other studies assessed the application and accuracy of malaria diagnosis by mRDT among pregnant women in Nigeria. Both sensitivity and specificity of mRDTs were $75 \%$ and $25 \%$ respectively (14) and therefore they were far lower than recommended values of sensitivity and specificity $(95 \%, 90 \%)$ respectively (6)(7). Another study examined the performance of mRDTs among HIV positive individuals people in Nigeria and the overall sensitivity of mRDT was $58 \%$ while the specificity was $97 \%$ and it was recommended to monitor quality of mRDTs for malaria diagnosis among HIV co- infected persons (15). Furthermore, a comparative study where they were comparing the performance of three brands of mRDTs namely SD Bioline, Paracheck and Acon, it was found that their sensitivity and specificity were : SD Bioline (86.3\%, 99.6\%); Paracheck (50\%, 97.7\%) and Acon $(66.7 \%, 100 \%)$, respectively and they recommended that mRDTs quality should be strongly monitored during transportation and storage process(16). Similarly, a longitudinal study conducted in Tanzania in 2011, in which accuracy and impact of mRDTs were being assessed, sensitivity of mRDT was found to be $88.6 \%$ while specificity of mRDTs was $88.2 \%(17)$. These sensitivity and specificity values were high, yet they were lower than recommended values of sensitivity and specificity(6)(7). It was argued that the low values of mRDTs (low sensitivity and specificity) were largely influenced by fever and parasites density. The authors recommended that the use of mRDTs should be coupled with supportive supervision in order to improve treatment of both malaria and non- malaria fevers and prevent the waste of anti-malaria drugs for malaria false positives 
patients (18).Other studies have shown that false-positive for results for mRDTs occur because the Plasmodium falciparum histidine rich protein

2 (pfHRP2) antigen remains in the bloodstream for some months after an infection is cleared which in turn could lead to overtreatment and the misdiagnosis of the true cause of symptoms (19). False-negative (FN) results of mRDTs were found to be influenced by either parasite density which is below the mRDTs limit of detection typically in the range of 200 parasites/ $\mu \mathrm{L}(20)$ or non - plasmodium falciparum malaria which is not detected by commonly used pfHRP2based mRDTs or adverse storage conditions of mRDTs where they are denatured by heat or humidity (21).

In 2008,the Rwanda Ministry of Health (MOH) piloted and scaled up the mRDTs (by community health workers) as recommended by WHO's Roll Back Malaria program (source) in the most of the regions as a way to expand and strengthen malaria diagnostic capacity throughout the country(22). In Rwanda, Community health workers (CHWs) are the first point of contact with healthcare services for those with fever or a history of fever. Binome community health workers (a woman and a man) were trained to perform mRDT to all suspected malaria cases and provide anti-malaria drugs to symptomatic individuals in their villages(23). In 2018, a research was carried in kayonza district (Eastern province of Rwanda) and compared the performance of HRP-2 based mRDT and microscopy- based malaria test. This study found that the sensitivity of mRDT was $95.0 \%$ while specificity was found to be $59.2 \%$ among 264 suspected patients (24) and despite the extensive training of $\mathrm{CHWs}$, the observed value for the specificity was below the acceptable levels. Moreover, little is known about the accuracy and predictive value of $\mathrm{mRDT}$ in the diagnosis of malaria infection at community level where asymptomatic people are more likely to have lower parasitaemia than in clinical settings (Vivi Maketa et al. 2013)[1]. Therefore, this study aimed to respond to this knowledge gap through an assessment of the accuracy, sensitivity, specificity and predictive values of histidine rich protein-2 (RHP-2) based mRDTs malaria in ngoma district, eastern province of Rwanda by comparing mRDTs results performed by CHWs with malaria microscopic results performed by qualified laboratory technicians at health centers (HCs) in order to assure or safeguard the performance of mRDTs.

[1] Vivi Maketa et al. 2013. Accuracy of MRDT on the diagnosis of malaria infection in children under 5 years without fever in the health zone of Mont Ngafulal, a hyper endemic area for malaria in Kinshasa, Democratic Republic of Congo

\section{Method}

\subsection{Research Design and Target Population}

This was a cross-sectional prospective study. All patients self-reported to CHWs for malaria diagnosis from $22^{\text {nd }}$ April to $08^{\text {th }}$ July, 2021 in Ngoma district who were willing to participate in this study were included. Patients who had recovered from malaria within the past three weeks were not included in this study because it was revealed that malaria antigen can be found in blood for 3weeks after completion of treatment which could increase false positive results(17). Patients who were taking anti malaria drug were not included in this study.

\subsection{Sample size}

Sample size was calculated by using the statistical formula of Cochran (1963:75) for calculating sample size for infinite population was used. It says that for large population for which the variability in proportion is unknown, it is assumed that the maximum variability is equal to $50 \%$ ( $p=0.5$ ) and at $95 \%$ confidence

level with $\pm 5 \%$ precision, the determination of needed sample size is as follows:

$$
\mathrm{n}=\frac{z^{2} p(1-p)}{e^{2}}
$$

where critical value of desired confidence level $z=1.96$ the

$$
\mathrm{n}=\frac{1.96^{2} \times 0.5(1-0.5)}{0.05^{2}}=384
$$

desired level of precision $e=0.05$, proportion $p=0.5(25)$. Therefore, the desired sample size

By adding the rate of no

respondent of $10 \%$ (38), the needed sample size, $n$, becomes 422 malaria suspected patients. In the end only 420 malaria suspected patients were considered in this study.

\subsection{Study site}

The study was conducted in Ngoma district, one of the six high-malaria burden districts in eastern province of Rwanda which has the highest malaria prevalence of $11.11 \%$ (3)(4). The number of cases of malaria tripled in the eastern province of Rwanda (from 460,460 in 2013-2014 to 1.4 million in 20152016)(3). Ngoma district borders Kayonza and Rwamagana districts in north, Bugesera district in south-west, Kirehe district in south-est. it also borders the country of Burundi in south. It has an area of $861 \mathrm{~km}^{2}$ with 15 health centers (HCs).

\subsection{Materials and data collection procedures}

A total of 109 binomes (CHWs) were randomly selected to take a blood sample and performed mRDT. A sample for thick blood smear for malaria microscopic test was also taken by the $\mathrm{CHWs}$ and the slides were taken to health center (HC) for processing by qualified microscopists. At the HC, the thick blood smears were stained using a solution of $10 \%$ Giemsa and left for 30 minutes and then washed in buffered water of pH 7.2. The blood smears were examined using 100x high power fields (26) and malaria parasites density was counted by using the formula developed by WHO, where: parasites/ $\mu \mathrm{L}$ blood $=($ No of parasites counted $x 8000$ white blood cells $/ \mu \mathrm{L}$ ) / No of white blood cells counted (27). Results of malaria microscopic tests and mRDT results were collected using a data collection form created using Epi info. Laboratory technicians at HCs were trained by Kibungo referral hospital on how to diagnose malaria using microscopy and how to calculate malaria parasites density before the study and similarly 15 data collectors were trained on how to collect data by using a data collection form created in Epi info. Smart phones were used to collect all data. 


\subsection{Data analysis procedure}

A mRDT positive case was defined by the presence of $P$. falciparum or a pan positive test line while a mRDT negative case was defined by the absence of $P$. falciparum and a pan test line. Results of mRDT were compared with results of malaria microscopic test and accuracy, sensitivity, specificity positive and negative predictive value of mRDT (RHP-2 based) were presented in terms percentages. Cohen's Kappa test was performed in order to determine level of agreement between mRDT and malaria microscopic results. Sensitivity and specificity of mRDT (RHP-2 based) kits were compared to WHO recommended value which is greater $95 \%$ for sensitivity and value which is greater or equal to $90 \%$ for specificity.

\section{Results}

\subsection{Socio-demographic characteristics of the studied population.}

Among 420 patients who were considered in this research 234 (55.71\%) were females and 186

(44.29\%) were males. Minimum age was 18 years while maximum age was 91 years. Mean age was 34.32 years with standard deviation of 14.01 as shown in Table 1 below.

Table 1: Descriptive presentation of the studied population

\begin{tabular}{llc} 
Variables & Frequency $(\mathrm{n}=420)$ & Percentage \\
\hline Age group in years & & 32.38 \\
\hline $18-24$ & 136 & 46.19 \\
\hline $25-44$ & 194 & 17.14 \\
\hline $45-64$ & 72 & 4.29 \\
65 and above & 18 & 55.71 \\
\hline Gender & & 44.29 \\
\hline Female & 234 & 186 \\
\hline Male & & 49 \\
\hline
\end{tabular}

\subsection{Comparison of mRDT results with malaria microscopic results}

Malaria positivity was found to be $2.62 \%$ by using mRDT and $1.67 \%$ by using microscopy as shown in Table 2 . No cases of pan-positive were found during this study, all positive cases were due to P.falciparum. The average parasite density obtained by microscopy was 451.8 with a range of $40-1200$ parasites/uL

Table 2: Test positivity of mRDT and malaria microscopic tests

\begin{tabular}{lll} 
Variables & \multicolumn{1}{l}{ Frequency $(\mathrm{n}=420)$} & Percentage \\
\hline \multicolumn{2}{l}{ Malaria rapid diagnostic test (mRD) $)$} & results \\
\hline Negative & 409 & 97.38 \\
\hline Positive & 11 & 2.62 \\
\hline Malaria microscopic test results \\
\hline Negative & 413 & 98.33 \\
\hline Positive & 7 & 1.67
\end{tabular}

In this study, the malaria microscopic test was used as reference test. The frequencies of mRDT's false positive and negative results are summarized in Table 3.

Table 3: Comparison of mRDT results to malaria microscopic test results 


\begin{tabular}{lll} 
Variables & Frequency $(\mathrm{n}=420)$ & Percentage \\
\hline \multicolumn{2}{l}{ mRDT results status } \\
\hline True negative & 408 & 97.14 \\
\hline True positive & 6 & 1.42 \\
\hline False negative & 1 & 0.23 \\
\hline False positive & 5 & 1.16
\end{tabular}

In this study mRDT had a sensitivity of $85.71 \%, 98.78 \%$ specificity, negative predictive value was $99.75 \%$, positive predictive value was $54.54 \%$, and Cohen's Kappa test $\mathrm{K}$ was found to be 0.642 . One false negative mRDT was observed with a microscopic malaria parasite density of 1200 parasites/ $\mu$ l.

During this study, by using logistic regression as shown in table 4\& 5, it was found that having malaria parasite density which was equal or greater than $100 /$ $\mu \mathrm{l}$ and was significantly associated with mRDT's sensitivity $>95 \%$ with $\mathrm{P}-\mathrm{V}=0.006$ at $95 \% \mathrm{Cl}(2.6397342 .6845)$ and OR of 30.07 . This means that malaria parasites density which was equal or greater than $100 / \mu$ l was 30.07 times more associated with having sensitivity $>95 \%$ than malaria parasites density less than $100 / \mu \mathrm{l}$. However, specificity was not statistically associated with malaria parasites density.

Table 4: Association between sensitivity of mRDT and malaria parasites

\begin{tabular}{|c|c|c|c|c|}
\hline \multicolumn{5}{|c|}{ Sensitivity of mRDT } \\
\hline Variables & $>95 \%$ & $<=95 \%$ & Statistical tests & \\
\hline & (n: 392) & $(n=28)$ & cOR at $95 \% \mathrm{Cl}$ & P.value \\
\hline \multicolumn{5}{|l|}{ Malaria parasites density } \\
\hline Malaria parasites density $<100 / \mu \mathrm{l}$ & 391 & 26 & $\operatorname{Ref}(1)$ & \\
\hline Malaria parasites density $>=100 / \mu \mathrm{l}$ & 1 & 2 & $30.07(2.26-342.6)$ & 0.006 \\
\hline
\end{tabular}

Table 5: Association between specificity of mRDT and malaria parasites density

\begin{tabular}{|c|c|c|c|c|}
\hline \multirow[b]{2}{*}{ Variables } & \multicolumn{3}{|c|}{ Specificity of mRDT } & \\
\hline & $>=90 \%$ & $<90 \%$ & Statistical tests & \\
\hline & (n: 392) & $(n=28)$ & cOR at $95 \% \mathrm{Cl}$ & P.value \\
\hline \multicolumn{5}{|l|}{ Malaria parasites density } \\
\hline Malaria parasites density $<100 / \mu l$ & 389 & 28 & 1 & \\
\hline Malaria parasites density $>=100 / \mu \mathrm{l}$ & 3 & 0 & 1 & \\
\hline
\end{tabular}

\section{Discussion}

This study provided information concerning diagnostic accuracy of mRDT performed by CHWs in Ngoma district. In this study, malaria positivity by using mRDT was slightly higher than malaria positivity by using microscopy. This finding agreed with several studies conducted by Ekom et al, Oliver et al and Mohamed et al(28)(9)(13) which reported higher malaria prevalence of $75 \%, 30.3 \%$ and $52.67 \%$ by using mRDTs compared to malaria prevalence of $60 \%$, $24.4 \%$ and $40.05 \%$ reported by using malaria microscopic test respectively.

In this study, mRDT had a sensitivity of $85.71 \%$ which was below the sensitivity recommended by WHO (>95\%) and a specificity of $98.78 \%$ which is within the recommended WHO value (6). The observed sensitivity of $85.71 \%$ is higher than other sensitivities found in different studies: Isa et al , Ekom et al and Rose et al found sensitivities of $40.3 \%, 75 \%, 50 \%, 66 \%$ (12) (28) (16), but lower than that found in other studies ( Niyibizi et al, Vyankatesh et al and Phoebe et al.) (24) (29)(17) demonstrating the wide range of mRDT's sensitivities. A low mRDT sensitivity can be a problem for patient care given that some patients who require an antimalarial treatment (the false negatives) will be missed. Indeed, in this study mRDT sensitivity $>95 \%$ was found to be associated with malaria parasites density equal or greater than $100 / \mu \mathrm{l}$, a finding that is in agreement with what was found by Bisoffi et al and Nwajei et al (30) (11). This limitation of the current mRDTs led to the development of newer generation of highly-sensitive mRDTs aimed at increasing the capability of mRDTs to detect low parasite density infections(31)(32). In addition to the parasite density, several other factors are known to affect the sensitivity of mRDTs including storage and transportation conditions which influence denaturation of mRDT kits and adherence to the protocol by the end user (Matthew et al, Rennie et al, Francois et al) (33)(34)(35). In this study, association between sensitivity and skills of end user, physical storage and transportation conditions was not investigated. 
The specificity of $98.78 \%$ identified in this present study was higher than other specificity found in other studies by Niyibizi et al, Anthony et al, Henry et al and Isa et al of $59.2 \%, 89 \%, 97 \%$ and $89.6 \%$ respectively(24) (11)(15) (12). This satisfactory specificity was not associated with malaria parasite density and is very important because it helps to differentiate other malaria-like illness from malaria and health care provider will be able to administer the right medications. In this present study, positive predictive value and negative predictive value were $54.54 \%$ and $99.75 \%$ respectively, comparing them to another study conducted in Nigeria, positive predictive value and negative predictive value were $81.8 \%$, and $56.5 \%$, respectively, Negatives predictive values seem to be nearly equal but positive predictive values are slightly different(12). This very low positive predictive value (54.54\%) reported in this present study was due to high false positive results of mRDT. False positive results of mRDT were found to be associated with high level of rheumatoid factors in patients (36) and the persistence of HRP-2 antigens in blood for up to 61 days after completion of treatment(19) which is another limitation of mRDTs. However, in the bigger scheme of things, treatment of false positive cases is less detrimental compared to lack of treatment of the false negatives although it contributes to overuse of antimalarials and appropriate treatment of missed non-malaria cases. Therefore, a confirmatory test is needed in case of doubtful positive result of mRDT.

Overall, the accuracy of mRDTs in this study was $98.57 \%$ implying that mRDT kits used in this study gave correct results either positive or negative at the level of $98.57 \%$ and a Cohen's Kappa test $k$ is equal to 0.642 , meaning that there was a good concordance with microscopy. Given that majority of malaria cases are diagnosed at the community lever by CHWs using mRDTs, these results demonstrates that the use of mRDTs by CHWs is appropriate and help increase the trust of population toward health care service provided by CHWs. However, continued supportive supervision and quality assurance of mRDTs is important to ascertain the quality of case management of malaria.

\title{
5. Conclusion
}

Overall, the accuracy of mRDTs in this study was acceptable and results were well correlated with microscopy results. This implies that the use of mRDTs by CHWs in Ngoma district is appropriate. The continued supportive supervision and quality assurance of mRDTs is important to ascertain the quality of case management of malaria at the community especially given that majority of malaria cases are managed at the community.

\author{
Abbreviations \\ CHWs \\ Community health workers \\ FN \\ False-negative \\ $\mathrm{HC}$ \\ Health center \\ mRDTs \\ Malaria rapid diagnostic tests \\ $\mathrm{MOH}$ \\ Ministry of Health \\ pfHRP2 \\ Plasmodium falciparum histidine rich protein 2.
}

\section{Declarations}

\section{Ethics approval and consent to participate}

Before starting data collection, ethical clearances were sought from Institute Review Board of University of Rwanda/ College of Medicine and Health Science and Kibungo referral hospital independent ethic committee. Before patients provided blood samples, they signed consent forms voluntarily. In order to ensure confidentiality of patients who provided samples, names of patients were removed from data set and every patient was represented by his/ her unique code auto generated by Epi info. In order to ensure the security of data set, it was protected with a password and stored in a laptop protected with a password.

\section{Consent for publication}

Not applicable

\section{Competing interests}

The authors declare that they have no competing interests

\section{Availability of data and materials}

The dataset used and analyzed during the current study is available from the corresponding author on reasonable request.

\section{Funding}


This study was fully funded by African Field Epidemiology Network (AFENET). The funding body had no role in the study design, collection, data analysis, data interpretation, or drafting of the manuscript.

Authors' Contributions: Conceptualization and Methodology, M.H., J. H., S. M. U., J. O., And L.W.N., Data collection, M.H, Data analysis, M.H and V.N, Writing, L.W.N., M.H., J. H, S. M. U., Supervision, J.H., S.M.U., J. O., S.U and V.N. All authors have read and approved the manuscript.

\section{Acknowledgements}

The authors thank Prof Joseph NTAGANIRA, Mr. Albert NGAGIJIMANA, Dr Theoneste NTAKIRUTIMANA, Mr. NIYOYITA Jean Claude, Mrs. Peace KINANI, Mr. Samuel RWUNGANIRA, African Field Epidemiology Network (AFENET), Rwanda Field Epidemiology and Laboratory Training, Kibungo Referral Hospital, patients, community health workers, laboratory technicians in health centers and at Kibungo referral hospital and supervisors of community health workers in health centers.

\section{References}

1. SERVICES VC, Cooperative M of PHCR of G. FINAL NATIONAL MALARIA PROGRAMME STRATEGIC PLAN 2015-2020 [Internet]. Guyana; 2018. Available from: https://docs.bvsalud.org/biblioref/2018/07/906525/arbo-guy-005.pdf

2. WHO. World malaria report 2020 [Internet]. 2020. Available from: https://www.who.int/publications/i/item/9789240015791

3. USAID. PRESIDENT'S MALARIA INITIATIVE RWANDA Malaria Operational Plan FY 2018 [Internet]. 2018. Available from: https://reliefweb.int/sites/reliefweb.int/files/resources/fy-2018-rwanda-malaria-operational-plan.pdf

4. Rudasingwa G, Cho S II. Determinants of the persistence of malaria in Rwanda. Malar J [Internet]. 2020;19(36):1-10. Available from: https://doi.org/10.1186/s12936-020-3117-z

5. WHO. Protocol for the Performance Evaluation of Malaria Rapid Diagnostic Tests for WHO Prequalification Assessment [Internet]. Geneve, Switzerland; 2020. Available from: https://www.who.int/diagnostics_laboratory/evaluations/alter/180814_protocol_pqdx_317_malaria_rdts_v1.0.pdf

6. WHO. New Perspectives Malaria Diagnosis [Internet]. Geneva; 2000. Available from: https://www.who.int/tdr/publications/documents/malariadiagnosis.pdf

7. Thompson S. Helpdesk Report: Malaria and Rapid Diagnostic Tests [Internet]. UK; 2012. Available from: http://www.heart-resources.org/wpcontent/uploads/2012/08/Malaria-and-Rapid-Diagnostic-Tests-August-2012.pdf

8. Kozycki CT, Umulisa N, Rulisa S, Mwikarago El, Musabyimana JP, Habimana JP, et al. False - negative malaria rapid diagnostic tests in Rwanda: impact of Plasmodium falciparum isolates lacking hrp2 and declining malaria transmission. Malar J [Internet]. 2017;16(123):1-11. Available from: https://malariajournal.biomedcentral.com/track/pdf/10.1186/s12936-017-1768-1.pdf

9. Watson OJ, Sumner KM, Janko M, Goel V, Winskill P, Slater HC, et al. False-negative malaria rapid diagnostic test results and their impact on communitybased malaria surveys in sub-Saharan Africa. BMJ Glob Heal [Internet]. 2019;4(e001582):1-9. Available from: https://www.ncbi.nlm.nih.gov/pmc/articles/PMC6666813/pdf/bmjgh-2019-001582.pdf

10. Simon IN, Malau MB, David MY, Emile NJ. Performance of Four Malaria Rapid Diagnostic Tests (RDTs) in the Diagnosis of Malaria in North Central Nigeria. Int J Infect Dis Ther [Internet]. 2020;5(4):106-11. Available from: http://www.sciencepublishinggroup.com/journal/paperinfo? journalid=280\&doi=10.11648/j.jijidt.20200504.11

11. Nwajei Al, Centre FM. Sensitivity and specificity of malaria rapid diagnostic test (mRDT CareStat TM) compared with microscopy amongst under five children attending a primary care clinic in southern Nigeria. African J Prim Heal Care Fam Med [Internet]. 2020; Available from: https://www.ncbi.nlm.nih.gov/pmc/articles/PMC7380062/pdf/PHCFM-12-2212.pdf

12. Abdulkadir I, Rufai HA, Ochapa SO, Malam MS, Garba BI. Malaria rapid diagnostic test in children: The Zamfara, Nigeria experience. Niger Med J [Internet]. 2015;56(4):278-82. Available from: https://www.ncbi.nlm.nih.gov/pmc/articles/PMC4697217/

13. Bah MS. The Relationship Between Malaria Status in Under-five Children and Some Household Demographic, Socioeconomic and Environmental Factors Associated with the Disease in Sierra Leone [Internet]. Georgia State University; 2020. Available from: https://core.ac.uk/download/pdf/322920575.pdf

14. Edem EN, Mbong EO, Hussain S. Accuracy of Malaria Diagnosis by RDT using Microscopy as gold standard amongst Pregnant Women Attending Antenatal Clinic in Eket. Rem Publ LLC [Internet]. 2020;2(1):1-4. Available from: https://www.longdom.org/open-access/accuracy-of-malaria-diagnosisby-microscopy-and-rdt-methods-amongst-pregnant-women-attending-antenatal-clinic-in-eket.pdf

15. Jegede FE. Evaluation of standard diagnostic rapid test kits for malaria diagnosis among HIV patients in Kano, Nigeria. Afr J Lab Med [Internet]. 2018;7(1):1-8. Available from: https://www.ncbi.nlm.nih.gov/pmc/articles/PMC6295795/pdf/AJLM-7-698.pdf

16. Ilesanmi RI, Olalubi OA, Adetunde OT, llesanmi AO, Effedua H, Amoo AO. Comparative assessment of malaria rapid diagnostic tests (RDT) in Ibadan, Nigeria. MalariaWorld J [Internet]. 2017;8(17):1-7. Available from: https://malariaworld.org/sites/default/files/mwjournal/article/MWJ2017_8_17.pdf

17. Mbabazi P, Hopkins H, Osilo E, Kalungu M, Byakika-kibwika P, Kamya MR. Accuracy of Two Malaria Rapid Diagnostic Tests (RDTS) for Initial Diagnosis and Treatment Monitoring in a High Transmission Setting in Uganda. Am Soc Trop Med Hyg Accuracy [Internet]. 2015;92(3):530-6. Available from: https://www.ncbi.nlm.nih.gov/pmc/articles/PMC4350543/pdf/tropmed-92-530.pdf

18. Ishengoma DS, Francis F, Mmbando BP, Lusingu JPA, Magistrado P, Alifrangis M, et al. Accuracy of malaria rapid diagnostic tests in community studies and their impact on treatment of malaria in an area with declining malaria burden in north-eastern Tanzania. Malar J [Internet]. 2011;10(176):1-13. Available from: https://www.ncbi.nlm.nih.gov/pmc/articles/PMC3145609/pdf/1475-2875-10-176.pdf 
19. Dalrymple U, Arambepola R, Gething PW, Cameron E. How long do rapid diagnostic tests remain positive after anti - malarial treatment ? Malar J [Internet]. 2018;17(228):1-14. Available from: https://doi.org/10.1186/s12936-018-2371-9

20. Zimmerman PA, Howes RE. Malaria diagnosis for malaria elimination. Curr Opin Infect Dis [Internet]. 2015;28(5):446-54. Available from: https://journals.Iww.com/co-infectiousdiseases/Fulltext/2015/10000/Malaria_diagnosis_for_malaria_elimination.7.aspx

21. Albertini A, Lee E, Coulibaly So, Sleshi M, Faye B, Mationg ML, et al. Malaria rapid diagnostic test transport and storage conditions in Burkina Faso, Senegal, Ethiopia and the Philippines. Malar J [Internet]. 2012;11(406):1-9. Available from:

https://malariajournal.biomedcentral.com/track/pdf/10.1186/1475-2875-11-406.pdf

22. Misbah G, Habtu M, Mochama M, Kansiime C, Asiimwe B. Factors Associated with Malaria Test Preference among Households in Rubavu District, Rwanda: A Cross-sectional Study. J Med Diagnostic Methods [Internet]. 2017;6(4):6-11. Available from: https://www.longdom.org/open-access/factorsassociated-with-malaria-test-preference-among-households-inrubavu-district-rwanda-a-crosssectional-study-2168-9784-1000261.pdf

23. Habimana A, Harerimana A, Asingizwe D, Nyandwi T, Njunwa KJ. Community Health Workers ' Knowledge, Attitudes and Practices about Malaria Prevention in Gicumbi District, Rwanda. Rwanda J Med Heal Sci [Internet]. 2016;3(1):27-35. Available from: https://www.ajol.info/index.php/rj/article/view/147018

24. Niyibizi JB, Gatera EK. Diagnostic Performance between Histidine-Rich Protein 2 (HRP-2), a Rapid Malaria Diagnostic Test and Microscopic-Based Staining Techniques for Diagnosis of Malaria. J Trop Med [Internet]. 2020;2020:2-7. Available from: https://downloads.hindawi.com/journals/jtm/2020/5410263.pdf

25. UNIVERSITY OF FLORIDA. Using Published Tables Using Formulas To Calculate A Sample Size Using A Census For Small Populations. In: Determining Sample Size [Internet]. p. 1-5. Available from: https://www.tarleton.edu/academicassessment/documents/samplesize.pdf

26. World Health Organization. MALARIA MICROSCOPY Quality Assurance Manual. Version 2 [Internet]. Geneva, Switzerland; 2016. Available from: https://www.who.int/docs/default-source/documents/publications/gmp/malaria-microscopy-quality-assurance-manual.pdf?sfvrsn=dfe54d47_2

27. World Health Organization. Malaria parasite counting [Internet]. 2016. Available from: https://www.who.int/publications/i/item/HTM-GMP-MM-SOP-09

28. Edem EN, Mbong EO, Hussain S. Accuracy of Malaria Diagnosis by RDT using Microscopy as gold standard amongst Pregnant Women Attending Antenatal Clinic in Eket. Ann Clin Immunol Microbiol. 2020;2(1):1-4.

29. Vyankatesh T. Anchinmane RTS. A Review of Malaria Diagnostic Tools: Microscopy and Rapid Diagnostic Test. ASIAN J Med Sci [Internet]. 2010;2:75-9. Available from:

https://www.researchgate.net/publication/273266189_A_Review_of_Malaria_Diagnostic_Tools_Microscopy_and_Rapid_Diagnostic_Test/link/5ef1219d2C

30. Bisoffi Z, Gobbi F, Van Den Ende J. Rapid diagnostic tests for malaria. Am Soc Microbiol. 2014;348(1):66-78.

31. Acquah FK, Donu D, Obboh EK, Bredu D, Mawuli B, Amponsah JA, et al. Diagnostic performance of an ultrasensitive HRP2 - based malaria rapid diagnostic test kit used in surveys of afebrile people living in Southern Ghana. Malar J [Internet]. 2021;20(125):1-11. Available from: https://doi.org/10.1186/s12936-021-03665-7

32. Medina AC, Tobo A, Posada M, Campillo A, Gonza IJ, Ding X. Performance of a highly sensitive rapid diagnostic test (HS-RDT) for detecting malaria in peripheral and placental blood samples from pregnant women in Colombia. PLoS One. 2018;13(8):1-14.

33. Boyce MR, Menya D, Turner EL, Laktabai J, Meara WPO. Evaluation of malaria rapid diagnostic test (RDT) use by community health workers: a longitudinal study in western Kenya. Malar J [Internet]. 2018;17(206):1-11. Available from: https://doi.org/10.1186/s12936-018-2358-6

34. Rennie W, Phetsouvanh R, Lupisan S, Vanisaveth V, Hongvanthong B, Phompida S, et al. Minimising human error in malaria rapid diagnosis: clarity of written instructions and health worker performance. Trans R Soc Trop Med Hyg. 2007 Jan;101(1):9-18.

35. Kiemde F, Tahita MC, Bonko A, Mens PF, Tinto H, Hensbroek MB Van, et al. Implementation of a malaria rapid diagnostic test in a rural setting of Nanoro, Burkina Faso : from expectation to reality. Malar J [Internet]. 2018;1-7. Available from: https://doi.org/10.1186/s12936-018-2468-1

36. Lee J, Jang W, Cho H, Kim Y, Han T, Yun G, et al. False-Positive Results for Rapid Diagnostic Tests for Malaria in Patients with Rheumatoid Factor. J Clin Microbiol [Internet]. 2014;52(10):3784-7. Available from: https://www.ghdonline.org/uploads/FP_RDT_zjm3784.pdf 